\title{
Use of veno-arterial extracorporeal membrane oxygenation in a case of tracheal injury repair in a patient with severe relapsing polychondritis
}

\author{
Anne-Sophie Laliberte, Christine McDonald, Tom Waddell, Kazuhiro Yasufuku \\ Division of Thoracic Surgery, Toronto General Hospital, University of Toronto, Toronto, Canada \\ Correspondence to: Anne-Sophie Laliberte, MD, FRCSC. Division of Thoracic Surgery, Toronto General Hospital, University of Toronto, Toronto, \\ Canada. Email: anne-sophie.laliberte@outlook.com.
}

\begin{abstract}
Tracheobronchial malacia occurs in $50 \%$ of patients with relapsing polychondritis (RP), and is often managed with stent insertion. While severe complications have been described after silicone tracheal stent insertion, there are few reports describing tracheal injury in patients with RP. We present a case of tracheal perforation secondary to Dumon ${ }^{\circledR}$ stent manipulation in a patient with RP. The tracheal injury was successfully repaired with a silicone Y-stent inserted via right thoracotomy using veno-arterial extracorporeal membrane oxygenation (VA-ECMO) for ventilatory support. It is safe and feasible to introduce a silicone Y-stent through a thoracotomy for a tracheal trauma in combination with VA-ECMO support.
\end{abstract}

Keywords: veno-arterial extracorporeal membrane oxygenation (VA-ECMO); tracheal injury; tracheal stent

Submitted May 22, 2017. Accepted for publication Sep 19, 2017.

doi: $10.21037 /$ jtd.2017.09.110

View this article at: http://dx.doi.org/10.21037/jtd.2017.09.110

\section{Introduction}

Relapsing polychondritis (RP) is a rare autoimmune disease characterised by inflammation of cartilage and is associated with tracheobronchial malacia (1). Respiratory complications account for significant morbidity and are associated with a poor prognosis (1). Stent insertion for tracheomalacia secondary to RP can be considered when patients fail to respond to medical therapy, but can lead to serious complications (2-4).

We describe a case of tracheal perforation secondary to Dumon ${ }^{\circledR}$ stent manipulation in a patient with tracheomalacia in the context of severe RP. The use of veno-arterial extracorporeal membrane oxygenation (VA-ECMO) allowed for successful tracheal repair via right thoracotomy.

\section{Case presentation}

A 55-year-old male with a history of RP, chronic uveitis and spondyloarthritis treated with prednisone and diclofenac presented with symptomatic tracheomalacia. In 2013, a 14-mm Dumon ${ }^{\circledR}$ stent with a length of $6 \mathrm{~cm}$ was inserted via rigid bronchoscopy. Adalimumab was initiated, and he was stable for 3 years.

He was followed with annual bronchoscopy to assess stent patency, and gradually developed granulation tissue at the distal end of the stent with increasing collapsibility of the airway beyond the stent. In May 2016, he developed recurrent pneumonias. The decision was made to exchange the existing stent with a longer stent to improve the integrity of the distal airway.

In July 2016, he underwent a planned rigid bronchoscopy and stent exchange in the operating room under general anesthetic. A flexible bronchoscopy was performed through an laryngeal mask airway. There was granulation tissue just distal to the stent and significant distal malacia.

An 8.5 -mm rigid bronchoscope was inserted to remove the tracheal stent. Forceps were used to grasp the stent; however, removal was not possible due to adhesions. After several attempts, a decision was made to leave the original stent in situ. The granulation tissue was debrided with forceps and the stent was advanced to provide better 


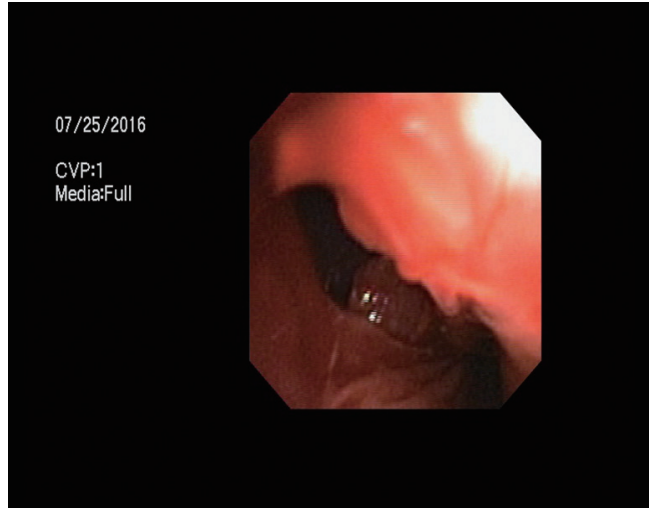

Figure 1 Flexible bronchoscopic image of the posterior tracheal wall laceration at the level of the carina.

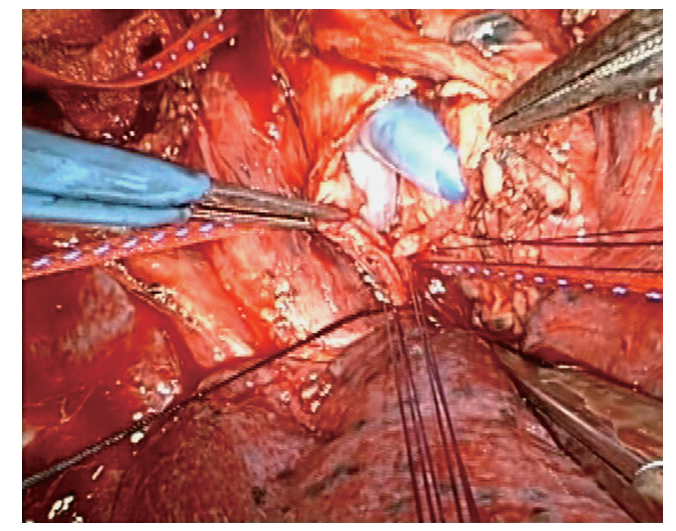

Figure 2 Left main bronchus and carinal repair. The endotracheal tube passes through the right arm of the Y-stent to allow for ventilation of the right lung.

support to the distal trachea.

When the patient awoke, he was stridorous with hypoxemia. He developed subcutaneous emphysema. Tracheal trauma was suspected. The patient was reanesthetised to evaluate for tracheal injury. The stent was successfully removed. A tracheal injury proximal to the left main bronchus was identified (Figure 1).

Multiples attempts were made to deploy a Y-stent through a Novatech Dumon Stent Insertion rigid bronchoscope to cover the tear. A $15-\mathrm{mm}$ diameter Dumon ${ }^{\circledR}$ Y-stent was used by cutting the left to $4.5 \mathrm{~cm}$ and the right arm to $2.5 \mathrm{~cm}$ based on bronchoscopic findings and CT measurement. The stent was difficult to place, and migrated through the posterior membrane of the trachea.

The patient became difficult to ventilate and was intubated with a size 5 Phycon armored endotracheal tube. Peripheral VA-ECMO was initiated and 5,000 units of heparin was administered prior to cannulation. A 15-French arterial cannula (Maquet) and 21-French venous cannula (Biomedicus, Medtronic) were inserted in the right femoral artery and vein. Flows varied between 3.4 and 4.5 litres per minute (Bio-pump, Medtronic). Adequate oxygenation was achieved with ECMO, and total cannulation time was $5 \mathrm{~h}$.

A right posterolateral thoracotomy was performed. An injury in the membranous portion of the left main bronchus extending to the carina was identified. Crossfield ventilation using an additional size 5 armored Phycon ${ }^{\circledR}$ endotracheal tube in the left main bronchus was performed.

An incision was made in the membranous portion of the distal trachea in order to insert a $14-\mathrm{mm}$ Dumon ${ }^{\circledR}$ Y-stent. The proximal end of the stent was introduced into the distal end of the endotracheal tube. The left arm of the Y-stent was inserted into the left main bronchus and the right arm into the right main bronchus. Corner stitches of 4-0 PDS were placed in the distal left main bronchus (Figure 2). Interrupted 4-0 PDS sutures were used to close the distal trachea. A water seal test was negative. Ventilation through the Y-stent was easy and bronchoscopy was performed to confirm stent positioning.

The patient remained hemodynamically stable and was decannulated from VA-ECMO. The endotracheal tube was changed to a 6.5 armored Phycon tube positioned above the Y-stent. The patient was transferred to the intensive care unit intubated and remained on pressure support ventilation for 5 days to facilitate pulmonary toileting.

On August 1st, he was extubated. He had difficulty clearing secretions and was urgently re-intubated 3 days later after a severe desaturation event. Daily bronchoscopy was performed to clear secretions from the stent and distal airways. We believe that the proximal trachea was collapsing above the stent, leading to dyspnea and failure of secretion clearance. The decision was made to perform an open tracheostomy and insert a size 7 armored tracheal tube (Bivona ${ }^{\circledR}$ ) to support the upper airway. Daily bronchoscopy was continued until the patient was able to manage the secretions via self-suctioning. The patient was discharged home with a fenestrated cuffless 6 Shiley ${ }^{\mathrm{TM}}$ tracheostomy tube.

The hospital stay totalled 43 days. Adalimumab was held. On the final day, he developed signs of a RP flare with an inflamed painful ear. Prednisone and adalimumab were resumed.

\section{Discussion}

RP can lead to severe tracheobronchial malacia. Endotracheal 
support via stent insertion can be lifesaving in select patients (1). Because RP is a progressive disease, multiple airway manipulations may be required over time. This presents a challenge as the anatomy may become distorted $(3,4)$. In our case manipulation of a Dumon ${ }^{\circledR}$ stent caused an injury localized at the membranous portion of the left main bronchus, which extended to the carina. This procedure should be avoided in the presence of adhesion and frail airway.

The Dumon ${ }^{\circledR}$ stent has been proven to be safe and effective, complications include coughing, obstruction, migration and granulation (5). The injury in this case was likely secondary to the severity of the patient's RP, with poor tracheal integrity.

Our first attempt to manage the tracheal injury was to cover the injured area using a Dumon ${ }^{\circledR}$ Y-stent. Endotracheal stents have been used successfully as a treatment for iatrogenic tracheal injury (6).

After several failed attempts to introduce the stent into the tracheal lumen, we elected to do a thoracotomy to repair the tracheal injury. Posterior wall repair has been described after tracheal injury during stent insertion (2). To our knowledge, this is the first reported case of tracheal repair via thoracotomy using ECMO in a patient with RP. The challenge in RP is repairing a trachea with no structural integrity and managing the malacia in the unstented portion of the airway thereafter.

Tracheal repair via right thoracotomy would have been impossible without the use of VA-ECMO for respiratory support. VA-ECMO was used in conjunction with crosstable ventilation to permit adequate oxygenation during the tracheal repair. The conventional indications of VAECMO are severe respiratory failure, severe cardiac failure or refractory cardiac arrest (7). More recently, VA-ECMO has been used as an alternative to in-field ventilation for complex tracheobronchial surgery (8). Our case adds to the literature of tracheobronchial repair facilitated by VAECMO in benign tracheal disease.

The challenging post-operative course was mainly attributed to RP. Because the Y-stent did not span the length of the trachea, the upper portion of the trachea was collapsing due to malacia. This was addressed by performing a tracheostomy to support the upper airway and facilitate secretion clearance.

\section{Conclusions}

It is safe and technically feasible to introduce a silicone Y-stent through a thoracotomy with respiratory support using VA-ECMO for the management of tracheal trauma.

\section{Acknowledgements}

None.

\section{Footnote}

Conflicts of Interest: The authors have no conflicts of interest to declare.

Informed Consent: Written informed consent was obtained from the patient for publication of this manuscript and any accompanying images.

\section{References}

1. Rafeq S, Trentham D, Ernst A. Pulmonary manifestations of relapsing polychondritis. Clin Chest Med 2010;31:513-8.

2. Lochowski MP, Szlachcińska A, Kozak J. Left mainstem bronchial laceration with perforation to right pleural cavity as complication after dynamic stent insertion. Wideochir Inne Tech Maloinwazyjne 2014;9:286-8.

3. Chapron J, Wermert D, Le Pimpec-Barthes F, et al. Bronchial rupture related to endobronchial stenting in relapsing polychondritis. Eur Respir Rev 2012;21:367-9.

4. Mitilian D, Gonin F, Sage E, et al. From relapsing polychondritis to extracorporeal membrane oxygenation. J Thorac Cardiovasc Surg 2013;146:e49-51.

5. Dumon JF. A dedicated tracheobronchial stent. Chest 1990;97:328-32.

6. Yamamoto S, Endo S, Endo T, et al. Successful silicon stent for life-threatening tracheal wall laceration. Ann Thorac Cardiovasc Surg 2013;19:49-51.

7. Tramm R, Ilic D, Davies AR, et al. Extracorporeal membrane oxygenation for critically ill adults. Cochrane Database Syst Rev 2015;1:CD010381.

8. Lang G, Ghanim B, Hötzenecker K, et al. Extracorporeal membrane oxygenation support for complex tracheobronchial procedurest. Eur J Cardiothorac Surg 2015;47:250-5; discussion 256.

Cite this article as: Laliberte AS, McDonald C, Waddell T, Yasufuku K. Use of veno-arterial extracorporeal membrane oxygenation in a case of tracheal injury repair in a patient with severe relapsing polychondritis. J Thorac Dis 2017;9(11):E1002-E1004. doi: 10.21037/jtd.2017.09.110 